\title{
Live cross exchange of plasma in normothermic perfusion to extend limb viability
}

Erin Guy and Emre Gok, MD*

University of Michigan, Ann Arbor, MI, 48104

\section{Introduction}

The current standard of care for preserving disarticulated limbs prior to transplantation is static cold storage (SCS). Limb preservation by SCS is limited at 4-6 hours. Ex-situ perfusion (ESP) is an alternative to SCS that preserves limb viability beyond 4-6 hours by mimicking normal physiologic functioning. Metabolite-waste equilibrium is a key factor in determining the limb's viability and predicting the overall success of the vascularized composite allograft. Plasma exchange (PE) may be a valuable addition to ESP circuits. PE from a live paracorporeal source might serve to remove aetiological factors from the limb preservation circuit and remedy circulatory deficiencies. The purpose of this study was to develop an ex-situ perfusion system, under near normal physiologic conditions, that integrated plasma exchange, to evaluate its effect on limb viability and transplantation in a rodent model.

\section{Method}

Near-normothermic conditions $\left(25-28^{\circ} \mathrm{C}\right)$ were established for the ESP system. The two circuits, limb and PE, were primed with Plasmalyte acellular solution. Perfusate consisted of leukocyte-depleted rat blood collected from 4 Lewis strain rat donors to achieve adequate hemoglobin levels $(\mathrm{HgB}>8 \mathrm{~g} / \mathrm{dL})$. Perfusate was then pushed from a roller pump $(0.5 \mathrm{~mL} / \mathrm{min})$ and filtered through a Hemocor HPH Jr. hemoconcentrator in the limb circuit. The PE circuit also used a Hemocor HPH Jr. hemoconcentrator. Perfusate was controlled at a rate of $36 \mathrm{~mL} / \mathrm{hr}$. Data collection involved limb perfusion pressure, oxygen consumption, and electrolyte concentration via hourly blood gas analyses; weight change of the limb prior to and following perfusion; and histopathology 3 months after the completed limb transplantation. Tissues samples were analyzed for injury scores using the full frame method.

\section{Results}

We maintained limb viability for six hours using ESP+PE, and performed successful allografts in the limb recipient, $\mathrm{n}=4$. Long term functional outcomes and viability scores are pending. We expect to see decreased injury scores in $\mathrm{ESP}+\mathrm{PE}$ groups compared to SCS, indicating a better functional outcome 3 months postoperatively.

\section{Discussion}

Continuous PE in normothermic ex-situ limb perfusion is a feasible method for prolonging the preservation of limbs. The removal of metabolic waste products and replacement via exchanged plasma reduces waste accumulation in the limb and improves the condition of the limb over six hours compared to SCS. Further studies are necessary to determine the maximal duration of ESP+PE and improve the mechanical circuit.

\footnotetext{
*To whom correspondence should be addressed: egok@med.umich.edu
} 